\title{
Discovery of two skin-derived dermaseptins and design of a TAT-fusion analogue with broad-spectrum antimicrobial activity and low cytotoxicity on healthy cells
}

\author{
Haohao Zhu ${ }^{1,2}$, Xiyan Ding ${ }^{1,2}$, Wei Li ${ }^{\text {corresp., }}{ }^{1}$, Tulin Lu ${ }^{1}$, Chengbang Ma corresp., ${ }^{2}$, Xinping Xi ${ }^{2}$, Lei Wang ${ }^{2}$, \\ Mei Zhou ${ }^{2}$, Roberta Burden ${ }^{2}$, Tianbao Chen ${ }^{2}$ \\ ${ }^{1}$ School of Pharmacy, Nanjing University of Chinese Medicine, Nanjing, China \\ 2 School of Pharmacy, The Queen's University Belfast, Belfast, United Kingdom \\ Corresponding Authors: Wei Li, Chengbang Ma \\ Email address: liwaii@126.com, c.ma@qub.ac.uk
}

Two novel peptides belonging to the dermaseptin family, namely DRS-CA-1 and DRS-DU-1, were encoded from cDNA libraries derived from the skin secretions of Phyllomedusa camba and Callimedusa (Phyllomedusa) duellmani. Both natural peptides are highlyconserved and exhibited high potency against wild-type Gram-positive, Gram-negative bacteria, yeast and antibiotic-resistant bacteria (MRSA and Pseudomonas aeruginosa) (MICs 4-8 $\mu \mathrm{M}$ ) with no obvious hemolytic activity. Collectively these results suggest that both peptides may have potential as novel antibiotics. Additionally, DRS-DU-1 exhibited selective cytotoxicity to tumor cells. The truncated analogue, DP-1 and TAT-fused DP-1 (namely DP-2) were subsequently synthesised. It showed that DP-1 had low antimicrobial activity, no hemolytic and cytotoxicity to tumor cells. However, DP-2 possessed strong antimicrobial activity and the similar selective, no obvious hemolytic activity and cytotoxicity on normal human cells, but enhanced cytotoxicity to tumor cells of DRS-DU-1. These findings indicate that the $\mathrm{N}$-terminus of the dermaseptins may contribute to their bioactivity, and that addition of the TAT peptide can improve biological activity. The results provide a new insight for designing novel peptide-based antimicrobial or anticancer agents with low hemolytic activity and cytotoxicity. 
1 Discovery of two skin-derived dermaseptins and design of a

2 TAT-fusion analogue with broad-spectrum antimicrobial 3 activity and low cytotoxicity on healthy cells

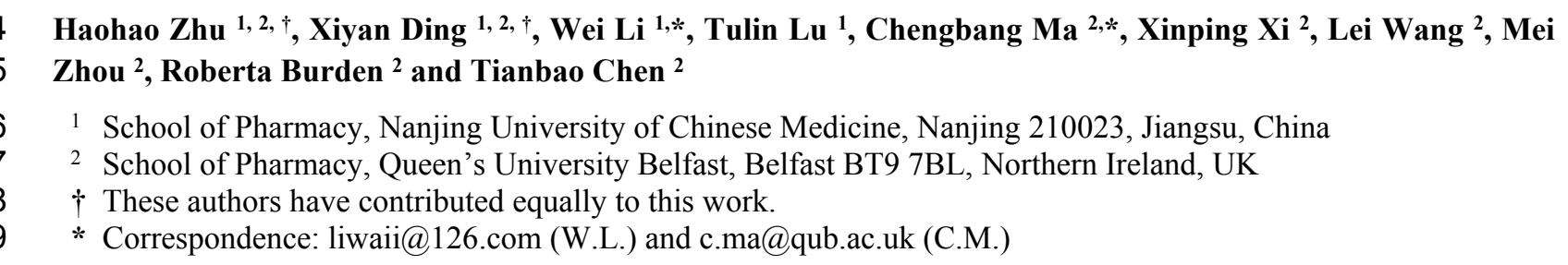

Abstract: Two novel peptides belonging to the dermaseptin family, namely DRS-CA-1 and DRS-DU-1, were encoded from cDNA libraries derived from the skin secretions of Phyllomedusa camba and Callimedusa (Phyllomedusa) duellmani. Both natural peptides are highly-conserved and exhibited high potency against wild-type Gram-positive, Gram-negative bacteria, yeast and antibiotic-resistant bacteria (MRSA and Pseudomonas aeruginosa) (MICs 4$8 \mu \mathrm{M}$ ) with no obvious hemolytic activity. Collectively these results suggest that both peptides may have potential as novel antibiotics. Additionally, DRS-DU-1 exhibited selective cytotoxicity to tumor cells. The truncated analogue, DP-1 and TAT-fused DP-1 (namely DP-2) were subsequently synthesised. It showed that DP-1 had low antimicrobial activity, no hemolytic and cytotoxicity to tumor cells. However, DP-2 possessed strong antimicrobial activity and the similar selective, no obvious hemolytic activity and cytotoxicity on normal human cells, but enhanced cytotoxicity to tumor cells of DRS-DU-1. These findings indicate that the N-terminus of the dermaseptins may contribute to their bioactivity, and that addition of the TAT peptide can improve biological activity. The results provide a new insight for designing novel peptide-based antimicrobial or anticancer agents with low hemolytic activity and cytotoxicity.

\section{Introduction}

Due to the lack of efficacy of current antibiotics against increasing numbers of drug-resistant pathogens, antimicrobial peptides are considered as the compounds with the most potential to take over traditional antibiotics against drug-resistant bacteria [1]. Peptides with antimicrobial and antifungal activity have vital roles in the innate immune system, which constitutes the first line of defense against a wide range of animal-invading pathogens [2-3]. The skin secretions of 
33 frogs from all around the world have been proven to be a valuable source of these antimicrobial 34 peptides, with more than one thousand antimicrobial peptides with significant different structural features having been extracted and characterized [4-5].

Dermaseptins are the largest family of antimicrobial peptides identified from the skin secretions of Phyllomedusa species. Although they show some differences in their lengths, they are clearly related, given that almost all members are K-rich polycationic peptides, with a tryptophan residue at position 3 and a highly-conserved motif in the central or C-terminal region [6]. Peptides belonging to the dermaseptin family normally have two apparent separated lobes of hydrophobicity and a positively-charged electrostatic surface, resulting in the coil-to-helix transition upon association with lipid bilayers [7-8]. The dermaseptins usually have lytic activity and are lethal against Gram-positive and Gram-negative bacteria, fungi and protozoa at micromolar concentrations [9]. Despite dermaseptins having identical amino acid sequences, they show significant differences in their efficiency and cytolytic activities, which was thought to be independent of the envelope structure of bacteria [10-12]. The antimicrobial potency of dermaseptin S3 (from Phyllomedusa sauvagii) was not affected after shortening its chain length, the truncated $\mathrm{N}$-terminal domain still retained complete or even better lytic activity against some bacteria [13]. Besides, the truncated analogues, such as $\mathrm{K}_{4}-\mathrm{S} 4(1-13) \mathrm{a}$ and $\mathrm{K}_{4}-\mathrm{S} 4(1-15) \mathrm{a}$ from dermaseptin S4, exhibited even lower cytotoxic effect on erythrocytes than the parent peptide [14-15].

Although most dermaseptins were discovered by their antimicrobial activity, they were still reported to be effective against the cancer cells. Dermaseptin B2 and B3 (from Phyllomedusa bicolor) exhibited potent anti-proliferative effect on PC-3 cells, and dermaseptin B2 inhibited the tumor growth of PC-3 cells in vivo [16-17]. The further study showed that dermaseptin B2 could interact with glycosaminoglycan (GAGs) on the surface of PC-3 for cell internalization [18]. Besides, dermaseptin PH (from Pithecopus hypochondrialis), PD1 and PD2 (from Pachymedusa dacnicolor) were reported to exhibit cytotoxicity against several human cancer cell lines [19-20].

In this study, we report two novel dermaseptins, namely DRS-CA-1 and DRS-DU-1, that were discovered by molecular cloning from cDNA libraries derived from the skin secretion of Phyllomedusa camba and Callimedusa (Phyllomedusa) duellmani. The mature peptides were subsequently synthesized by solid-phase peptide synthesis and purified for functional assays. In 
63 addition, two analogues were designed based on the results to study the potential cytotoxicity to

64 tumor cells of dermaseptins.

65

66

67

68

69

\section{Materials and Methods}

\subsection{Specimen Biodata and Secretion Harvesting}

Adult specimens of $P$. camba and $C$. duellmani were obtained from commercial sources (PeruBiotech E.I.R.L., Lima, Peru). Before harvesting the skin secretion, all frogs were cultivated in the purpose-designed amphibian facility for at least four months, conditioned at 20$25{ }^{\circ} \mathrm{C}$ with $12 \mathrm{~h} / 12 \mathrm{~h}$ light/dark cycle and fed with multivitamin-loaded crickets three times per week. The defensive skin secretions were produced by stimulation the glands on the skin surface of frogs with gentle transdermal electrical stimulation (6V DC; $4 \mathrm{~ms}$ pulse-width; $50 \mathrm{~Hz})$ through platinum electrodes for two periods of 20 s duration. The study was performed according to the guidelines in the UK Animal (Scientific Procedures) Act 1986, project license PPL 2694, issued by the Department of Health, Social Services and Public Safety, Northern Ireland. Procedures had been vetted by the IACUC of Queen's University Belfast, and approved on 1st March, 2011.

\subsection{Molecular Cloning of the Dermaseptin peptide precursor-encoding cDNAs}

Five milligrams of crude lyophilized skin secretions were used to obtain polyadenylated mRNA using the Dynabeads ${ }^{\circledR}$ mRNA DIRECT ${ }^{\text {TM }}$ kit (Dynal Biotech Ltd, UK). cDNA library construction and primary cDNA amplification were performed with a BD SMART ${ }^{\mathrm{TM}}$ RACE cDNA amplification kit (BD Biosciences, UK) to give full-length prepro-peptide nucleic acid sequence data. The degenerate sense primer (S1; 5'ACTTTCYGAWTTRYAAGMCCAAABATG-3') $\quad(\mathrm{Y}=\mathrm{C}+\mathrm{T}, \quad \mathrm{W}=\mathrm{A}+\mathrm{T}, \quad \mathrm{R}=\mathrm{A}+\mathrm{G}, \quad \mathrm{M}=\mathrm{A}+\mathrm{C}$, $\mathrm{B}=\mathrm{T}+\mathrm{C}+\mathrm{G}$ ) applied in the RACE reactions were designed according to the highly conserved domain of the 5'-untranslated region of dermaseptin cDNAs from Phyllomedusa species. RACE products were subjected to gel analysis, and the detected bands were purified and cloned using the pGEM $^{\circledR}-\mathrm{T}$ Easy vector system (Promega, USA). The sequences were obtained from an ABI 3100 automated capillary sequencer.

\subsection{Reverse phase HPLC fractionation of crude skin secretion and amino-acid sequence} analysis of relevant peptides 
91

92

93

94

95

96

97

A further five milligrams of lyophilised skin secretion were dissolved in $0.5 \mathrm{ml}$ of $0.05 \%$ (v/v) trifluoroacetic acid (TFA)/water and clarified by centrifugation. The clear supernatant was subjected to reverse-phase HPLC on an analytical column (Jupiter C-5, $250 \mathrm{~mm} \times 10 \mathrm{~mm}$, Phenomenex, UK), eluted with a $0-80 \%$ linear gradient of acetonitrile containing $0.05 \%(\mathrm{v} / \mathrm{v})$ TFA in $240 \mathrm{~min}$ at a flow rate of $1 \mathrm{ml} / \mathrm{min}$. Absorbance was monitored at $214 \mathrm{~nm}$. The peptide mapping of two natural occurring peptides was conducted using MS/MS fragmentation sequencing against the cDNA encoding peptide precursors by LCQ electrospray ion-trap mass spectrometer (Thermo Fisher Scientific, San Francisco, CA, USA).

\subsection{Solid-Phase Peptide Synthesis}

Both the natural peptides and the analogs were chemically synthesized through the solidphase method with standard Rink amide resin and Fluorenylmethoxycarbonyl (Fmoc) chemistry in a PS4 automated solid-phase synthesizer (Protein Technologies, Inc, Tucson, AZ, USA). After cleavage from the resin and the protecting groups, the crude peptides were purified and analyzed by reverse-phase HPLC and MALDI-TOF mass spectrometry.

\subsection{Secondary Structure and Physicochemical Properties Prediction of the Peptides}

I-TASSER webserver was applied to predict the secondary structures of the synthetic peptides [21]. Physiochemical properties of the peptides were predicted by Heliquest and the helical wheel plots of the secondary structures were obtained from the helical wheel projections [22].

In addition, circular dichroism (CD) analyses were performed on the JASCO J815 Spectropolarimeter (JASCO Inc., USA). The measure range of each sample was 190-260 nm, and the parameters were set as follows: $0.5 \mathrm{~nm}$ data pitch, $1 \mathrm{~nm}$ bandwidth and $200 \mathrm{~nm} / \mathrm{min}$ scanning speed. The peptide samples were prepared with $10 \mathrm{mM}$ ammonium acetate buffer and $50 \%$ trifluoroethanol (TFE) in $10 \mathrm{mM}$ ammonium acetate buffer at a concentration of $100 \mu \mathrm{M}$. The percentage of the $\alpha$-helix structure was predicted by BESTSEL online software [23].

\subsection{Antimicrobial assays}

Gram-positive bacteria Staphylococcus aureus (NCTC10788), methicillin-resistant Staphylococcus aureus (MRSA) (NCTC12493), and Enterococcus faecalis (NCTC 12697); Gram-negative bacteria Escherichia coli (NCTC 10418), Pseudomonas aeruginosa (ATCC 
120

121

122

123

124

125

126

127

128

129

130

131

132

133

134

135

136

137

138

139

140

141

142

143

144

145

146

147

148

149

27853), and Klebsiella pneumoniae (ATCC 43816), and the yeast Candida albicans (NCYC 1467) were chosen to determine the antimicrobial activities of synthesized peptides through evaluating the minimum inhibitory concentration (MIC) and minimum bactericidal concentration (MBC). The microorganisms were cultured in Muller Hinton Broth (MHB, Oxoid, UK) medium and incubated in the orbital incubator (Stuart, UK) at $37^{\circ} \mathrm{C}$ overnight and then sub-cultured in a pre-warmed MHB medium until the bacteria reached their respective logarithmic growth phases. The sub-cultured suspension was diluted with fresh medium to a concentration of $5 \times 10^{5}$ colony forming units $(\mathrm{CFU}) / \mathrm{ml}$. The peptides with different concentrations were prepared in DMSO from $512 \mu \mathrm{M}$ to $1 \mu \mathrm{M}$. The MICs were determined in 96-well microtiter plates by mixing the peptides with bacteria, and 1\% DMSO in MHB was applied as the negative control. After an incubation of $20 \mathrm{~h}$, the growth of microorganisms was detected at a wavelength of $550 \mathrm{~nm}$ in a Synergy HT plate reader (Biolise BioTek EL808, Winooski, VT, USA). The MICs were determined as the lowest concentration of peptide where no apparent growth of the microorganism was detectable. Subsequently, $10 \mu$ of solution in each well was spotted on the Mueller Hinton Agar (MHA) plates. The MBC values were determined as the lowest concentration of peptide where no growth was observed on the MHA plate.

\subsection{Hemolysis assay}

Fresh defibrinated horse blood (TCS Biosciences Ltd, Buckingham, UK) was used to perform the hemolysis assay. The serum and erythrocytes were separated by centrifugation and the erythrocytes were collected and washed with PBS. Peptides in concentration gradients were incubated with $4 \%(\mathrm{v} / \mathrm{v})$ erythrocyte suspension at $37{ }^{\circ} \mathrm{C}$ for $2 \mathrm{~h} .1 \%$ DMSO and triton X-100 (Sigma Aldrich, St. Louis, MO, USA) was used as the negative and positive control, respectively. After centrifugation, $100 \mu \mathrm{l}$ supernatants were transferred to a 96-well plate and absorbance measured at $550 \mathrm{~nm}$ using a Synergy HT plate reader (BioTec, USA). Peptide concentration causing 50\% hemolysis of the red bloods cells $\left(\mathrm{HC}_{50}\right)$ was calculated [\% haemolysis $=(\mathrm{A}-\mathrm{A} 0) /(\mathrm{AX}-\mathrm{A} 0) \times 100$, where ' $\mathrm{A}$ ' is absorbance with peptides of different concentrations, 'A0' is absorbance with negative controls and 'AX' is absorbance with positive controls] to determine the hemolysis activities. The therapeutic index (TI) was calculated as $\mathrm{HC}_{50}$ divided by the geometric mean of the MIC values against relevant bacteria.

\subsection{Cell Lines and Cell Culture}


150

151

152

153

154

155

156

157

158

159

160

161

162

163

164

165

166

167

168

169

170

171

172

173

174

175

176

177

178

Human prostate carcinoma cell line, PC-3 (ATCC-CRL-1435), non-small cell lung cancer cell line, H157 (ATCC-CRL-5802), breast cancer cell line MDA-MB-435s (ATCC-HTB-129), neuronal glioblastoma cell line U251MG (ECACC-09063001) and breast cancer nontumorigenic mammary gland cell line, MCF-7 (ATCC-HTB-22) were utilized to screen the cytotoxicity of synthetic peptides. PC-3 and H157 cell lines were cultured in RPMI-1640 medium (Invitrogen, Paisley, UK), and the other cell lines were cultured with Dulbecco's Modified Eagle's medium (DMEM) (Sigma, St. Louis, MO, USA). Culture media was supplemented with 10\% fetal bovine serum (FBS) (Sigma, UK) and 1\% penicillin streptomycin solution (Sigma, UK). The human mammary epithelial cell line, HMEC-1 (ATCC-CRL-3243) was used to evaluate the cytotoxicity of the synthetic peptides against normal human cells, and cultured with MCDB131 medium (Gibco, Paisley, UK) with 10\% FBS, 10ng/ml EGF, 10mM LGlutamine and 1\% penicillin streptomycin. The selected cell lines were resuscitated and transferred into a $75 \mathrm{~cm}^{2}$ culture flask, and then incubated at $37^{\circ} \mathrm{C}$ atmosphere containing $5 \%$ $\mathrm{CO}_{2}$.

\subsection{Assessment of Antiproliferative Activity with the MTT Assay}

MTT cell viability assay was used to assess the proliferation and viability of different cell lines. After cell quantification, the cell suspension was mixed with pre-warmed medium to the final concentration of $5 \times 10^{3}$ cells in 96-well plates. After treating with serum-free medium, various concentrations of peptides were added and incubated for $24 \mathrm{~h}, 1 \%$ DMSO in related culture media was treated as the negative control. $10 \mu \mathrm{l}$ of MTT solution $(5 \mathrm{mg} / \mathrm{ml})$ (Sigma, UK) was added to each well and incubated for $4 \mathrm{~h}$. Supernatants were removed by syringe and $100 \mu \mathrm{l}$ of DMSO was used to resuspend the insoluble purple formazan crystals. The absorbance of each well was measured at $570 \mathrm{~nm}$ with the Synergy HT plate reader (BioTek, Winooski, VT, USA), data were analysed to obtain the mean and standard error of responses. The dose-response curves were constructed using a "best-fit" algorithm and then the half maximal inhibitory concentrations $\left(\mathrm{IC}_{50}\right)$ were calculated through the data analysis package provided in GraphPad Prism 6 (GraphPad Software, USA).

\section{Results}

3.1. Identification of Two Dermaseptins from Their Skin Secretion and Bioinformatic Analyses of 
Novel Peptides

Two novel peptides, namely DRS-CA-1 and DRS-DU-1, were encoded from two different cDNA libraries derived from $P$. camba and C. duellmani, which were the first dermaseptin discovered from respective frog species. The nucleotide and translated open-reading frame consisting of 76 amino acid sequences are shown in Figure 1. Both contain 16-residue signal peptide regions, followed by a 16-residue Glu-rich acidic peptide spacer and 28-residue mature peptide with typical dermaseptin sequence, connected by two -KR-propeptide convertase processing sites. The extension (-GEQ-) was also observed, of which G acted as a donor for Cterminal amidation of mature peptides. LC-MS analysis confirmed the presence of DRS-CA-1 and DRS-DU-1 in the skin secretion of $P$. camba and $C$. duellmani, respectively, possessing the post-translational modification of C-terminal amidation (Figure 2 A\&B). Alignment of the full length nucleic acid sequences and the open-reading frame sequences of the two cloned precursors are shown in Figure 3. Both nucleotide and amino acid sequences demonstrated high degree of similarity (92\%) between two biosynthetic precursors. Specifically, there was only one residue different in the signal peptides and three residues different in the mature peptides between the two moieties. A BLAST search in the Nonredundant Protein Sequence Database showed that several dermaseptins isolated from different frog species share a high degree of identity of amino acid sequences with the two dermaseptins identified herein and their openreading frame sequences also exhibited remarkable similarities in the acidic peptides (Figure 3). The cDNA sequences of two dermaseptin precursors have been deposited in the GenBank Database under the accession codes, MF955846 and MF955847.

\subsection{Design, Synthesis, Physicochemical Properties and Secondary Structure Prediction of}

\section{Peptides and their Analogues}

Considering the length of dermaseptin, N-terminal of 13-mer truncated mimetic peptide, DP-1, was designed following the previous study [24]. In the meantime, the TAT (GRKKRRQRRR) peptide was introduced at the N-terminal of DP-1 to improve its cellpenetration ability, namely DP-2. A Gly was added as a linker between TAT and DP-1. Both dermaseptins and the designed analogues were successfully synthesized and purified.

The predicted physicochemical properties and secondary structures of the peptides are shown in Table 1 and Figure 4, respectively. Both natural peptides had 3 positive net charges and 
209 possessed $\alpha$-helical secondary structure. Additionally, the calculated $\alpha$-helicity of the natural 210 peptides based on the CD spectra is $23.5 \%$ and $27.9 \%$, respectively. However, DP-1 had lower 211 degree of helicity as $16.1 \%$ though it had the same net charges and relatively higher 212 hydrophobicity and hydrophobic moment. Whilst, DP-2 contained the lowest helical content of $21311.4 \%$. Helical wheel plots of four peptides are shown in Figure 4C. The two natural peptides 214 formed the hydrophobic face, which are associated with antimicrobial activity of peptides. 215 However, both DP-1 and DP-2 revealed no uninterrupted hydrophobic face.

\subsection{Antimicrobial and Hemolytic Activities}

The $\mathrm{MIC}, \mathrm{MBC}$ and $\mathrm{HC}_{50}$ values of all the peptides obtained from antimicrobial and hemolysis assays are summarized in Table 2. The two natural peptides, DRS-CA-1 and DRSDU-1, showed the same MIC values $(4 \mu \mathrm{M})$ against $S$. aureus and E. coli and $C$. albicans. DRSDU-1 was 2-fold more potent against MRSA, E. faecalis, $K$. pneumoniae and P. aeruginosa than DRS-CA-1. The MBC values of two natural peptides against 7 tested microorganisms were two or four-fold higher than respective MICs. No obvious hemolysis activity was detected at the MIC or MBC concentrations except against E. faecalis .

The two designed peptides also exhibited broad spectrum antimicrobial activities against the 7 tested microorganisms, though the potency of DP-1 was much lower. However, the antimicrobial activities of DP-2 were restored comparing to DP-1. Additionally, the antimicrobial potency of DP-2 on E. faecalis was higher than the natural peptides.

\subsection{Anti-Proliferative Effects of the Peptides}

The anti-proliferative $\mathrm{IC}_{50}$ values of the natural peptides and designed peptides determined by MTT assay are summarized in Table 3. DRS-CA-1 and DP-1 were found to possess no obvious anti-proliferative activity against all five human cancer cell lines and normal human cells. DRS-DU-1 had selective activity against $\mathrm{H} 157$ and PC-3 cells, with $\mathrm{IC}_{50}$ values of $8.43 \mu \mathrm{M}$ and $21.6 \mu \mathrm{M}$, respectively. Although DP-1 had no activity against all cancer cell lines, the activity of DP-2 was improved considerably, with the $\mathrm{IC}_{50}$ values of $3.21 \mu \mathrm{M}$ and $6.75 \mu \mathrm{M}$ against H157 and PC-3 cells respectively. In addition, the cytotoxicity against normal human cell line (HMEC-1) of DRS-DU-1 ( $\mathrm{IC}_{50}$ of 53.75) was eliminated. The $\mathrm{IC}_{50}$ values of DP-1 and DP-2 against HMEC-1 are both greater than $100 \mu \mathrm{M}$. 


\section{Discussion}

240 Many bacteria have developed resistance against conventional antibiotics that has pressed

241 researchers to discover novel antibacterial agents [25]. In recent years, antimicrobial peptides

242 isolated from amphibian skin secretions have become a hot topic in both academic and industrial

243 drug discovery research. It is believed that most antimicrobial peptides act on the cell membrane,

244 and can distinguish target from host cells through differences in fluidity and the negative charge

245 density of their membranes. Such properties make it unlikely for the bacterial pathogens to

246 become resistant to antimicrobial peptides [26]. However, studies reported that the resistance of

247 bacteria to AMPs has emerged recently, by producing positively-charged molecules on the

248 membrane, and pumping AMPs out of cells [27-28]. Therefore, discovery and design more

249 effective antimicrobial peptide seems to be urgent and important.

250 In this study, we aimed to discover novel bioactive peptides from two rarely studied species

251 of the Phyllomedusidae family, P. camba and C. duellmani. As a result, two novel dermaseptin

252 peptides were successfully identified. The nucleotide and translated open-reading frame

253 sequences of the peptides have almost identical signal peptide sequences, the same prepropeptide

254 processing site and amino donor. Furthermore, they differ by only two amino acids in the acidic 255 peptide sequence and three residues in the mature peptides. Through the NCBI-BLAST search, it 256 is interesting to note that the two novel precursors not only exhibit high homology to the 257 different types of antimicrobial peptides from the Phyllomedusidae frogs, but also to other antimicrobial peptides from the Hylidae tree frogs. More than $80 \%$ identity were shared in the signal peptide domain between the two dermaseptins precursors and the antimicrobial peptides derived from the Hylidae frogs, including dermatoxin, medusin, caerin, cruzioseptin and phylloseptin [29-33]. This is the reason of the evolution from the ancestors that were selected by the surroundings to form diverse bioactive peptides for their unique defense system [34].

DRS-CA-1 and DRS-DU-1 exhibited strong antimicrobial activity against Gram-positive and Gram-negative bacteria and fungi with no obvious hemolytic activity, and almost the same proportion of polar residues and nonpolar residues as well as the $\alpha$-helical structure. The positive net charge of antimicrobial peptides had been widely accepted as the key factor to help the 
267 peptide combine with the negatively-charge bacteria cell surface. Besides, the hydrophobic 268 surface of the peptides in the $\alpha$-helical structure could ensure the peptides permeabilize the 269 membrane. Although resistance against antimicrobial peptides have emerged, this bacterial270 killing mechanism of dermaseptin is distinctly different from conventional antibiotics, which 271 makes it promising in relation to overcoming antibiotic resistance problems [35]. Comparing to 272 the two most famous dermaseptins, dermaseptin B2 and S4, both natural peptides demonstrated 273 potent antimicrobial activities, though dermaseptin B2 showed strongly inhibitory effect on $E$. 274 coli $[13,36]$. As the studies indicated, more cationicity of dermaseptin could improve the 275 antimicrobial activity as well as the spectrum, which is consistent with study of introduce of Lys 276 at positions 7 and 14 of dermaseptin S4 that remarkably increased the antimicrobial activity 277 against $P$. aeruginosa [37]. Additionally, the study of truncated analogues demonstrated that the 278 integrity of N-terminus is essential for possessing the antimicrobial activity while reducing the 279 haemolysis within certain degree [13]. Therefore, we assumed that the 13-mer N-terminal 280 truncated analogue, DP-1, will retain the antimicrobial potency of the patent peptides, but will 281 become less hemolytic. Although the DP-1 had the same ratio of polar/nonpolar residues, higher 282 hydrophobic moment and same positive net charge, it might not form the proper $\alpha$-helical

283

284

285

286

287

288

289

290

291

292

293

294

295

296

297 structure resulting in significant reduction of its antimicrobial activity against all microorganisms. The data is different comparing to the previous study of the structure-activity relationship of dermaseptin S4 [13], in which peptides with same physico-chemical properties usually have similar potency. This phenomenon indicates the importance of peptide's secondary structure in designing short antimicrobial peptides. Interestingly, when the TAT peptide was added, its antimicrobial activity was enhanced remarkably with reduced hemolytic activity. Considering their structural difference, this can be explained by the increased positive charges, which can enhance the interaction with the negative charge bacterial membranes. However, research has revealed that a high positive charge could lead to an increased hemolytic activity and a loss of antimicrobial potency [38], which is not corresponding with the results. We hypothesize that TAT peptide associated the attachment to the surface of bacteria, which subsequently allow the helical region of DP-1 segment to permeabilize the cell membrane.

Although dermaseptin has been reported to induce necrosis in PC-3 cancer cells, there are researches showing that dermaseptin could enter the cytoplasm and the nucleus $[18,39]$. We also noticed that the sensitivity to dermaseptin varies due to different type of cancer cells. On the 
298

299

300

301

302

303

304

305

306

307

308

309

310

311

312

313

314

315

316

317

318

319

320

321

322

323

324

325

sensitive cells, PC-3, researches indicated that the internalization mechanism may be initiated through the interaction with negatively-charged glycosaminoglycan (GAGs) [18]. Therefore, the disappearance of cytotoxic activity of DP-1 could be the shorter sequence and lower helicity that decreases the permeability against cell membrane, as well as the affinity to cancer cells for the GAGs mediated internalization. However, the restore of cytotoxicity activity of DP-2 suggests that fused-TAT improved the affinity of DP-2 to the cell membrane and initiated the interaction with GAGs.

On the other hand, the cytotoxicity of antimicrobial peptides on normal mammalian cells is mostly associated with the hydrophobicity and helicity of those peptides. The typical membrane lytic peptide, melittin, possesses an average helicity of $70 \%$ at membrane interfaces [40]. When reviewing the researches of different dermaseptins, we found that the increase of helicity not only improved antimicrobial and cytotoxic effect, but also increased the cytotoxicity on both normal cell lines and erythrocytes $[12,20]$. For instance, that dermaseptin-PH showed a $\alpha$ helicity of $35 \%$ may be related to the high degree of cytotoxicity on HMEC-1 [12]. The presence of cytotoxicity on both cancer cells and HMEC-1 of DRS-DU1 than DRS-CA1 could be also speculated by the higher $\alpha$-helicity. Additionally, researches of truncated dermaseptin analogues also indicates that the integrity of $\alpha$-helix and hydrophobic domain is important for the bioactivity of dermaseptin [39]. Moreover, that the effects of DP-2 on HMEC-1 and erythrocytes were remained mild is consistent with the hypothesis of GAGs involved internalization as proportion of GAGs is larger than which is on the healthy cells.

In summary, two novel dermaseptins were deduced from the skin secretion of $P$. camba and C. duellmani with broad-spectrum antimicrobial activity even against drug-resistant strains (MRSA and Pseudomonas aeruginosa) with low hemolytic activity, which makes them promising in the treatment of multidrug-resistant bacteria. This study revealed the importance of the TAT peptide in the design of antimicrobial or anti-proliferative cancer agents through enhancing their biological function. All of these provide a new idea for designing novel peptidebased antimicrobial or anti-proliferative agents. 
327

328

329

330

331

332

333

334

335

336

337

338

339

340

341

342

343

344

345

346

347

348

349

350

351

352

\section{References}

[1] R. Li, H. Wang, Y. Jiang, Y. Yu, L. Wang, M. Zhou, T. Chen, C. Shaw, A novel Kazal-type trypsin inhibitor from the skin secretion of the Central American red-eyed leaf frog, Agalychnis callidryas, Biochimie. 94 (2012) 1376-1381.

[2] D. Shi, Y. Luo, Q. Du, L. Wang, M. Zhou, J. Ma, T. Chen, C. Shaw, A novel bradykininrelated dodecapeptide (RVALPPGFTPLR) from the skin secretion of the fujian large-headed frog (Limnonectes fujianensis) exhibiting unusual structural and functional features, Toxins. 6 (2014) 2886-2898.

[3] T. Chen, L. Li, M. Zhou, P. Rao, B. Walker, C. Shaw, Amphibian skin peptides and their corresponding cDNAs from single lyophilized secretion samples: identification of novel brevinins from three species of Chinese frogs, Peptides. 27 (2006) 42-48.

[4] V. Erspamer, P. Melchiorri, G.F. Erspamer, P.C. Montecucchi, R. De Castiglione, Phyllomedusa skin: a huge factory and store-house of a variety of active peptides, Peptides. 6 (1985) 7-12.

[5] L. Azevedo-Calderon, A. Sliva, P. Ciancaglini, R.G. Stabeli, Antimicrobial peptides from Phyllomedusa frogs: from biomolecular diversity to potential nanotechnologic medical applications, Amino acids. 40 (2011) 29-49.

[6] P. Nicolas, C. El Amri, The dermaseptin superfamily: a gene-based combinatorial library of antimicrobial peptides, Biochim. Biophys. Acta. 1788 (2009) 1537-1550.

[7] T. Chen, L. Tang, C. Shaw, Identification of three novel Phyllomedusa sauvagei dermaseptins (sVI-sVIII) by cloning from a skin secretion-derived cDNA library, Regul. Peptides. 116 (2003) 139-146.

[8] L. Wang, M. Zhou, A. McClelland, A. Reilly, T. Chen, R. Gagliardo, B. Walker, C. Shaw, Novel dermaseptin, adenoregulin and caerin homologs from the Central American red-eyed leaf frog, Agalychnis callidryas, revealed by functional peptidomics of defensive skin secretion, Biochimie. 90 (2008) 1435-1441. 
353 [9] Y. Shin, R.W. Moni, J.E. Lueders, J.W. Daly, Effects of the amphiphilic peptides 354 mastoparan and adenoregulin on receptor binding, G proteins, phosphoinositide breakdown, cyclic AMP generation, and calcium influx, Cell. Mol. Neurobiol. 14 (1994) 133-157.

[10]L. Rivas, J.R. Luque-Ortega, D. Andreu, Amphibian antimicrobial peptides and Protozoa: Lessons from parasites, BBA-Biomembranes. 1788 (2009) 1570-1581.

358

359

360

361

362

363

364

365

366

367

368

369

370

371

372

373

374

375

376

377

378

379

[11]S. Navon-Venezia, R. Feder, L. Gaidukov, Y. Carmeli, A. Mor, Antibacterial properties of dermaseptin S4 derivatives with in vivo activity, Antimicrob. Agents. Ch. 46 (2002) 689694.

[12]L. Huang, D. Chen, L. Wang, C. Lin, C. Ma, X. Xi, T. Chen, C. Shaw, M. Zhou, Dermaseptin-PH: a novel peptide with antimicrobial and anticancer activities from the skin secretion of the south American orange-legged leaf frog, Pithecopus (Phyllomedusa) hypochondrialis, Molecules. 22 (2017) 1805.

[13]I. Kustanovich, D. E. Shalev, M. Mikhlin, L. Gaidukov, A. Mor, Structural requirements for potent versus selective cytotoxicity for antimicrobial dermaseptin S4 derivatives, J. Biol. Chem. 277 (2002) 16941-16951.

[14]C. M. Shepherd, H. J. Vogel, D. P. Tieleman, Interactions of the designed antimicrobial peptide MB21 and truncated dermaseptin S3 with lipid bilayers: molecular-dynamics simulations, Biochem. J. 370 (2003) 233-243.

[15]R. Feder, A. Dagan, A. Mor, Structure-activity relationship study of antimicrobial dermaseptin S4 showing the consequences of peptide oligomerization on selective cytotoxicity, J. Biol. Chem. 275 (2000) 4230-4238.

[16]H. van Zoggel, Y. Hamma-Kourbali, C. Galanth, A. Ladram, P. Nicolas, J. Courty, J. Delbé, Antitumor and angiostatic peptides from frog skin secretions, Amino Acids. 42(2012), 385395.

[17]H. van Zoggel, G. Carpentier, C. D. Santos, Y. Hamma-Kourbali, J. Courty, M. Amiche, J. Delbé, Antitumor and angiostatic activities of the antimicrobial peptide dermaseptin B2, PloS one. 7 (2012) e44351. 
380 [18]C. D. Santos, S. Hamadat, K. L. Saux, C. Newton, M. Mazouni, L. Zargarian, M. Amiche, 381 Studies of the antitumor mechanism of action of dermaseptin B2, a multifunctional cationic 382 antimicrobial peptide, reveal a partial implication of cell surface glycosaminoglycans, PloS one. 12 (2017) e0182926.

384

385

386

387

388

389

390

391

392

393

394

395

396

397

398

399

400

401

402

403

404

405

406

[19]L. Huang, D. Chen, L. Wang, C. Lin, C. Ma, X. Xi, M. Zhou, Dermaseptin-PH: A Novel Peptide with Antimicrobial and Anticancer Activities from the Skin Secretion of the South American Orange-Legged Leaf Frog, Pithecopus (Phyllomedusa) hypochondrialis, Molecules. 22 (2007) 1805.

[20]D. Shi, X. Hou, L. Wang, Y. Gao, D. Wu, X. Xi, C. Shaw, Two novel dermaseptin-like antimicrobial peptides with anticancer activities from the skin secretion of Pachymedusa dacnicolor, Toxins. 8 (2016) 144.

[21]A. Roy, A. Kucukural, Y. Zhang, I-TASSER: a unified platform for automated protein structure and function prediction, Nat. Protoc. 5 (2010) 725.

[22]R. Gautier, D. Douguet, B. Antonny, G. Drin, HELIQUEST: a web server to screen sequences with specific $\alpha$-helical properties, Bioinformatics. 24 (2008) 2101-2102.

[23]A. Micsonai, F. Wien, L. Kernya, Y. H. Lee, Y. Goto, M. Réfrégiers, J. Kardos, J. Accurate secondary structure prediction and fold recognition for circular dichroism spectroscopy, $\mathrm{P}$. Natl. Acad. Sci. USA. 112 (2015) E3095-E3103.

[24]R. Feder, A. Dagan, A. Mor, Structure-activity relationship study of antimicrobial dermaseptin S4 showing the consequences of peptide oligomerization on selective cytotoxicity, J. Biol. Chem. 275 (2000) 4230-4238.

[25]H. C. Neu, The crisis in antibiotic resistance, Science. 257 (1992) 1064-1074.

[26]M. R. Yeaman, N. Y. Yount, Mechanisms of antimicrobial peptide action and resistance, Pharmacol. Rev. 55 (2003) 27-55.

[27]H. S. Joo, C. I. Fu, M. Otto, Bacterial strategies of resistance to antimicrobial peptides, Phil. Trans. R. Soc. B. 371 (2016) 20150292.

[28]D. L. Andersson, D. Hughes, J. Z. Kubicek-Sutherland, Mechanisms and consequences of 
407

408

409

410

411

412

413

414

415

416

417

418

419

420

421

422

423

424

425

426

427

428

429

430

431

432

433

bacterial resistance to antimicrobial peptides, Drug. Resist. Update, 26 (2016) 43-57.

[29]T. Chen, B. Walker, M. Zhou, C. Shaw, C. Dermatoxin and phylloxin from the waxy monkey frog, Phyllomedusa sauvagei: cloning of precursor cDNAs and structural characterization from lyophilized skin secretion, Regul. Peptides. 129 (2005) 103-108.

[30]Y. Gao, D. Wu, L. Wang, C. Lin, C. Ma, X. Xi, C. Shaw, Targeted Modification of a Novel Amphibian Antimicrobial Peptide from Phyllomedusa tarsius to Enhance Its Activity against MRSA and Microbial Biofilm, Front. Microbiol. 8 (2017) 628.

[31]Y. Gao, D. Wu, X. Xi, Y. Wu, C. Ma, M. Zhou, C. Shaw, Identification and characterisation of the antimicrobial peptide, phylloseptin-PT, from the skin secretion of Phyllomedusa tarsius, and comparison of activity with designed, cationicity-enhanced analogues and diastereomers, Molecules. 21 (2016) 1667.

[32]T.L. Pukala, T. Bertozzi, S.C. Donnellan, J.H. Bowie, K.H. Surinya, Y. Liu, M.J. Tyler, Host-defence peptide profiles of the skin secretions of interspecific hybrid tree frogs and their parents, female Litoria splendida and male Litoria caerulea, FEBS. J. 273 (2006) 3511-3519.

[33]C. Proano-Bolanos, M. Zhou, L. Wang, L.A. Coloma, T. Chen, C. Shaw, Peptidomic approach identifies cruzioseptins, a new family of potent antimicrobial peptides in the splendid leaf frog, Cruziohyla calcarifer, J. Proteomics. 146 (2016) 1-13.

[34]D. Vanhoye, F. Bruston, P. Nicolas, M. Amiche, Antimicrobial peptides from hylid and ranin frogs originated from a 150-million-year-old ancestral precursor with a conserved signal peptide but a hypermutable antimicrobial domain, FEBS. J. 270 (2003) 2068-2081.

[35]T.H. Lee, K. N Hall, M.I. Aguilar, Antimicrobial peptide structure and mechanism of action: a focus on the role of membrane structure, Curr. Top. Med. Chem. 16 (2016) 25-39.

[36]P. Joanne, C. Galanth, N. Goasdoué, P. Nicolas, S. Sagan, S. Lavielle, I. D. Alves, I. D, Lipid reorganization induced by membrane-active peptides probed using differential scanning calorimetry, BBA-Biomembranes. 1788 (2009) 1772-1781.

[37]Z. Jiang, A. I. Vasil, M. L. Vasil, R. S. Hodges, "Specificity determinants" improve 
434 therapeutic indices of two antimicrobial peptides piscidin 1 and dermaseptin S4 against the 435 gram-negative pathogens Acinetobacter baumannii and Pseudomonas aeruginosa, $436 \quad$ Pharmaceuticals. 7 (2014) 366-391.

437 [38]M. Dathe, H. Nikolenko, J. Meyer, M. Beyermann, M. Bienert, Optimization of the 438 antimicrobial activity of magainin peptides by modification of charge, FEBS. Lett. 501 $439 \quad$ (2001) 146-150.

440 [39]C. Auvynet, N. Seddiki, I. Dunia, P. Nicolas, M. Amiche, C. Lacombe, Post-translational 441 amino acid racemization in the frog skin peptide deltorphin $\mathrm{I}$ in the secretion granules of 442 cutaneous serous glands, Eur. J. Cell. Biol. 85(2006), 25-34.

443 [40] M. Andersson, J. P. Ulmschneider, M. B. Ulmschneider, S. H. White, Conformational states 444 of melittin at a bilayer interface, Biophys. J. 104(2013), L12-L14. 


\section{Table $\mathbf{1}$ (on next page)}

The predicted physicochemical properties and secondary structure of DRS-CA-1, DRSDU-1, DP-1, and DP-2

Data from physicochemical property prediction. 


\begin{tabular}{|c|c|c|c|c|c|}
\hline Peptides & Sequence & $\begin{array}{c}\alpha \text {-Helicity } \\
(\%)\end{array}$ & $\begin{array}{l}\text { Hydrophobicity } \\
\text { (H) }\end{array}$ & $\begin{array}{l}\text { Hydrophobic } \\
\text { moment }(\mu \mathrm{H})\end{array}$ & $\begin{array}{c}\text { Net } \\
\text { charge }\end{array}$ \\
\hline DRS-CA-1 & $\begin{array}{c}\text { ALWKDLLKNVGKAAGKAVLNK } \\
\text { VTDMVNQ.NH }\end{array}$ & 25.3 & 0.291 & 0.263 & +3 \\
\hline DRS-DU-1 & $\begin{array}{l}\text { ALWKSLLKNVGKAAGKAALNAV } \\
\text { TDMVNQ. } \mathrm{NH}_{2}\end{array}$ & 27.9 & 0.331 & 0.255 & +3 \\
\hline DP-1 & ALWKSLLKNVGKA.NH ${ }_{2}$ & 16.1 & 0.429 & 0.645 & +3 \\
\hline DP-2 & $\begin{array}{l}\text { GRKKRRQRRRGALWKSLLKNVG } \\
\text { KA.NH }{ }_{2} \\
\end{array}$ & 11.4 & -0.112 & 0.358 & +11 \\
\hline
\end{tabular}

1 
Table 2 (on next page)

Antimicrobial (MIC and $\mathrm{MBC}$ ) and hemolytic $\left(\mathrm{HC}_{50}\right)$ activity, and relative safety $(\mathrm{TI})$ of DRS-CA-1, DRS-DU-1, DP-1, and DP-2.

Data represent the mean of $\geq 3$ determinations 


\begin{tabular}{ccccc}
\hline \multirow{2}{*}{ Strains } & \multicolumn{4}{c}{ MIC/MBC $(\boldsymbol{\mu M})$} \\
\cline { 2 - 4 } & DRS-CA-1 & DRS-DU-1 & DP-1 & DP-2 \\
\hline S. aureus & $4 / 16$ & $4 / 16$ & $64 / 128$ & $8 / 8$ \\
MRSA & $8 / 32$ & $4 / 16$ & $128 / 128$ & $16 / 16$ \\
E. faecalis & $128 / 256$ & $64 / 128$ & $256 />512$ & $32 / 128$ \\
E. coli & $4 / 16$ & $4 / 16$ & $64 / 128$ & $4 / 8$ \\
P. aeruginosa & $8 / 32$ & $4 / 16$ & $128 / 256$ & $8 / 16$ \\
K. pneumoniae & $8 / 128$ & $4 / 64$ & $128 />512$ & $32 / 256$ \\
C. albicans & $4 / 16$ & $4 / 16$ & $32 / 64$ & $4 / 8$ \\
Horse Erythrocytes & 114.7 & 216.6 & $>512$ & $>512$ \\
(HC 50 & & & 13.93 & 147.13 \\
\hline TI (overall) & 21.73 & 54.15 & &
\end{tabular}

1 


\section{Table 3(on next page)}

$\mathrm{IC}_{50}$ of DRS-CA-1, DRS-DU-1, DP-1, and DP-2 against tested human cancer cells and human normal cell.

The cytotoxicity of dermaseptin B2 (DRSB2), dermaseptin PH (DRSPH), dermaseptin PD1 (DRSPD1) and dermaseptin PD2 (DRSPD2) obtained from published data were shown in the table for comparison. 


\begin{tabular}{ccccccccc}
\hline \multirow{2}{*}{ Cell lines } & \multicolumn{8}{c}{ IC $_{\mathbf{5 0}}(\boldsymbol{\mu M})$} \\
\cline { 2 - 9 } & DRS-CA- & DRS-DU- & DP- & DP- & DRSB & DRSP & DRSPD & DRSPD \\
& $\mathbf{1}$ & $\mathbf{1}$ & $\mathbf{1}$ & $\mathbf{2}$ & $\mathbf{2}$ & $\mathbf{H}$ & $\mathbf{1}$ & $\mathbf{2}$ \\
\hline HMEC-1 & $>100$ & 53.75 & $>100$ & $>100$ & NA & 4.85 & 36.35 & 27.28 \\
H157 & $>100$ & 8.43 & $>100$ & 3.21 & NA & 2.01 & NA & 6.43 \\
PC-3 & $>100$ & 21.6 & $>100$ & 6.75 & 2.17 & 11.8 & NA & 3.17 \\
MDA-MB- & $>100$ & $>100$ & $>100$ & $>100$ & $>10$ & 9.94 & NA & NA \\
435s & & & & & & & & \\
U251MG & $>100$ & $>100$ & $>100$ & $>100$ & NA & 2.36 & 15.08 & 13.43 \\
MCF-7 & $>100$ & $>100$ & $>100$ & $>100$ & NA & 0.69 & NA & NA \\
\hline
\end{tabular}

1 NA: Not tested. 


\section{Figure 1}

Translated amino acid sequence of the open-reading frame and nucleotide sequence of cloned CDNA encoding the biosynthetic precursor of DRS-CA-1 (A) from Callimedusa (Phyllomedusa) camba and DRS-DU-1 (B) from Phyllomedusa duellmani.

The putative signal peptides are marked with double line, mature peptides are marked with single line and stop codons are indicated by an asterisk.

*Note: Auto Gamma Correction was used for the image. This only affects the reviewing manuscript. See original source image if needed for review. 
A)

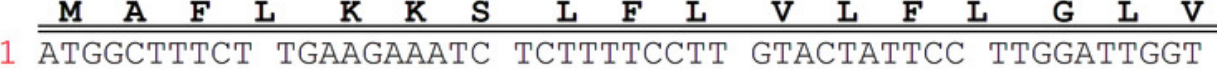
TACCGAAAGA ACTTCTTTAG AgAAAAgGAA CATGATAAgG AACCTAACCA $\begin{array}{llllllllllllllllll}\cdot & \mathrm{S} & \mathrm{L} & \mathrm{S} & \mathrm{I} & \mathrm{C} & \mathrm{E} & \mathrm{E} & \mathrm{E} & \mathrm{K} & \mathrm{R} & \mathrm{E} & \mathrm{N} & \mathrm{E} & \mathrm{E} & \mathrm{K} & \mathrm{E} & \mathrm{N}\end{array}$

51 TTCTCTTTCT ATCTGTGAAg AAGAGAAAA AgAAAATGAA GAGAAAGAAA AAGAGAAAGA TAGACACTTC TTCTCTTTTC TCTTTTACTT CTCTTTCTTT \begin{tabular}{lllllllllllllllll}
$\cdot$ & $\mathbf{Q}$ & $\mathbf{E}$ & $\mathrm{D}$ & $\mathrm{D}$ & $\mathbf{E}$ & $\boldsymbol{Q}$ & $\mathbf{S}$ & $\mathbf{E}$ & $\mathbf{E}$ & $\mathrm{K}$ & $\mathrm{R}$ & $\mathbf{A}$ & $\mathrm{L}$ & $\mathrm{W}$ & $\mathrm{K}$ & $\mathrm{D}$ \\
\hline
\end{tabular}

101 ATCAAGAAGA TGATGAGCAA AgtGAAGAgA AGAGAgCTCT GTGGAAAGAT TAGTTCTTCT ACTACTCGTT TCACTTCTCT TCTCTCGAGA CACCTTTCTA $\begin{array}{llllllllllllllllll}\text { L } & \text { L } & \text { K } & \text { N } & \text { V } & \text { G } & \text { K } & \text { A } & \text { A } & \text { G } & \text { K } & \text { A } & \text { V } & \text { L } & \text { N } & \text { K } & \text { V }\end{array}$

151 TTATTAAAAA ATGTAgGgAA AgCTGCAgGA AAAgCGgTTT TAAATAAAgT AATAATTTTT TACATCCCTT TCGACGTCCT TTTCGCCAAA ATTTATTTCA $\begin{array}{lllllllllll}\cdot & \mathrm{T} & \mathrm{D} & \mathrm{M} & \mathrm{V} & \mathrm{N} & \mathrm{Q} & \mathrm{G} & \mathrm{E} & \boldsymbol{Q} & \text { * }\end{array}$

201 TACTGATATG GTAAATCAAG GAGAGCAATA AAgTTAAGAA AATGTAAATT ATGACTATAC CATTTAGTTC CTCTCGTTAT TTCAATTCTT TTACATTTAA

251 TCATAACTCT AAGGAGCACA ATTATCAATA ATTGTAGGCA AACTTATATT AGTATTGAGA TTCCTCGTGT TAATAGTTAT TAACATCCGT TTGAATATAA

301 AAAGCATATT GAACAAAAAA AAAAAAAAAA AAAAAAAAAA TTTCGTATAA CTTGTTTTTT TTTTTTTTTT TTTTTTTTTT

B)

\begin{tabular}{lllllllllllllllll}
$\mathbf{M}$ & $\mathbf{A}$ & $\mathbf{F}$ & $\mathbf{I}$ & $\mathrm{K}$ & $\mathrm{K}$ & $\mathbf{S}$ & $\mathrm{L}$ & $\mathbf{F}$ & $\mathrm{L}$ & $\mathrm{V}$ & $\mathrm{L}$ & $\mathbf{F}$ & $\mathrm{L}$ & $\mathrm{G}$ & $\mathrm{L}$ & $\mathrm{V}$ \\
\hline
\end{tabular}

1 ATGGCTTTCA TTAAGAAATC TCTTTTCCTT GTACTATTCC TTGGATTGGT TACCGAAAgT AATTCTTTAG AgAAAAgGA CATGATAAgG AACCTAACCA $\begin{array}{lllllllllllllllllll}. & \mathrm{S} & \mathrm{L} & \mathrm{S} & \mathrm{I} & \mathrm{C} & \mathrm{E} & \mathrm{E} & \mathrm{E} & \mathrm{K} & \mathrm{R} & \mathrm{E} & \mathrm{N} & \mathrm{E} & \mathrm{E} & \mathrm{E} & \mathrm{N} & \mathrm{N}\end{array}$

51 TtCCCTTTCC ATCTGTGA AAgAgAAAAg AgAAAATGA GAAgAAAATA AAGGGAAAGG TAGACACTTC TTCTCTTTTC TCTTTTACTT CTTCTTTTAT

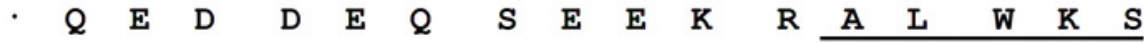

101 AtCAAGAAGA TGATGAgCAA AgTGAAGAgA AGAGAGCTCT GTGGAAATCT TAGTTCTTCT ACTACTCGTT TCACTTCTCT TCTCTCGAGA CACCTTTAGA \begin{tabular}{lllllllllllllllll}
$\mathrm{L}$ & $\mathrm{L}$ & $\mathrm{K}$ & $\mathrm{N}$ & $\mathrm{V}$ & $\mathrm{G}$ & $\mathrm{K}$ & $\mathrm{A}$ & $\mathrm{A}$ & $\mathrm{G}$ & $\mathrm{K}$ & $\mathrm{A}$ & $\mathrm{A}$ & $\mathrm{L}$ & $\mathrm{N}$ & $\mathrm{A}$ & $\mathrm{V}$ \\
\hline
\end{tabular}

151 TTATTAAAAA ATGTAgGgAA AgCTGCAGGA AAAgCGgCTT TAAATGCAGT AATAATTTT TACATCCCTT TCGACGTCCT TTTCGCCGAA ATTTACGTCA

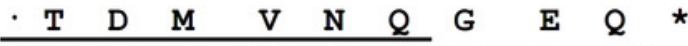

201 TACTGATATG GTAAATCAAG GAGAGCAATA AAgTTAAGAA AATGTAAATC ATGACTATAC CATTTAGTTC CTCTCGTTAT TTCAATTCTT TTACATTTAG

251 AAATTGCTCT AAGGAGCACA AATTAATCAA TCAATTGTTA ACTTAGCTTA TTTAACGAGA TTCCTCGTGT TTAATTAGTT AGTTAACAAT TGAATCGAAT

301 TATTAAAGCA TATTGAACCA AAAAAAAAAA AAAAAAAAAA ATAATTTCGT ATAACTTGGT TTTTTTTTTT TTTTTTTTTT 


\section{Figure 2}

Identification of (A) DRS-CA-1 and (B) DRS-DU-1 from the corresponding skin secretions. The retention times of DRS-CA-1 and DRS-DU-1 are indicated by arrows in the respective HPLC chromatograms

The spectra and the tables beside demonstrate the peptide mapping using SEQUEST algorithm. 

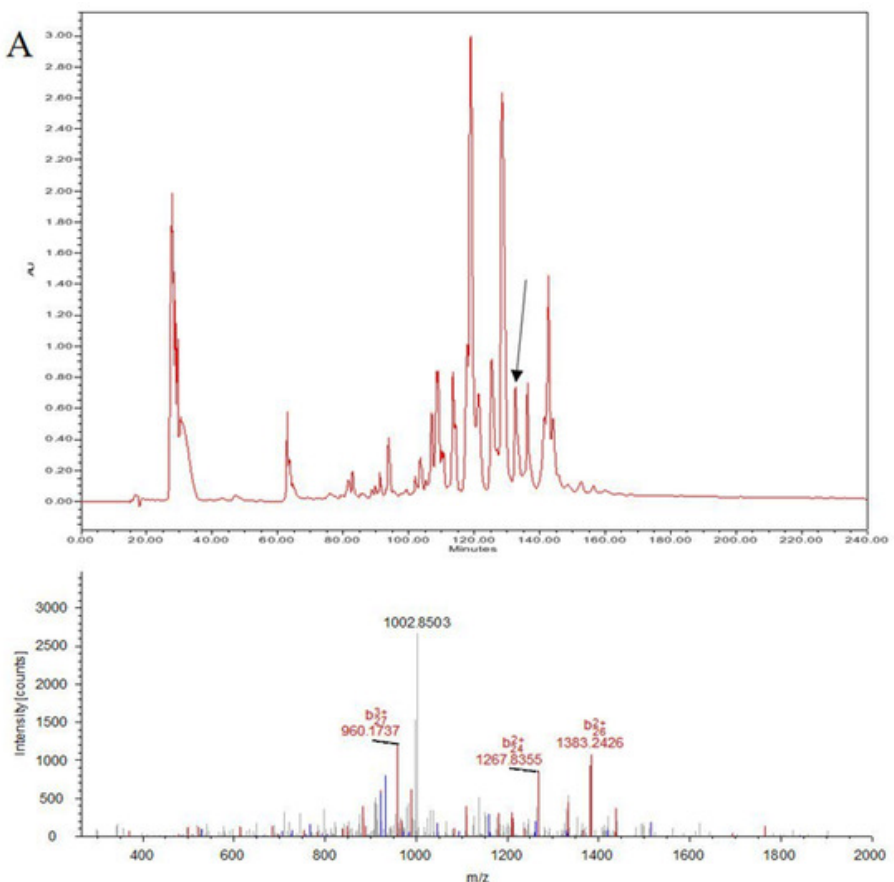

B
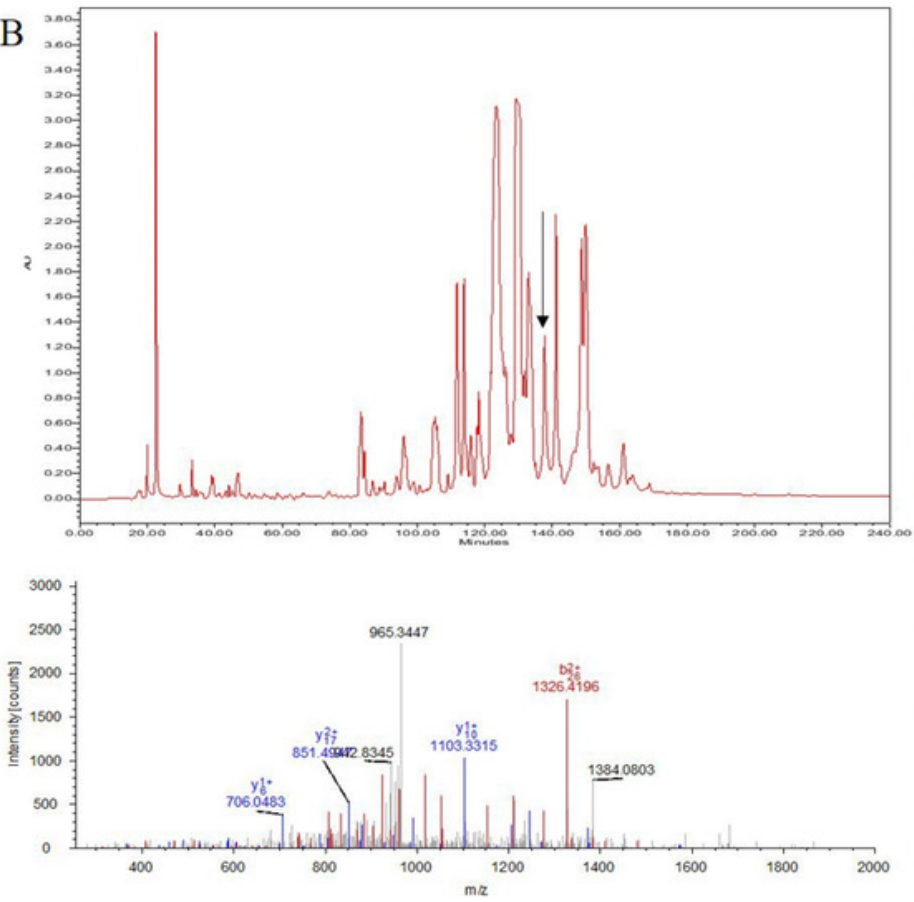

\begin{tabular}{|c|c|c|c|c|c|c|c|c|}
\hline$\# 1$ & $b(1+)$ & $b(2+)$ & $b(3+)$ & Seq. & $y(1+)$ & $y(2+)$ & $y(3+)$ & $\# 2$ \\
\hline 1 & 72.044 & 36.526 & 24.686 & A & & & & 28 \\
\hline 2 & 185.128 & 93.068 & 62.381 & L & 2952.6817 & 1476.8445 & 984.8988 & 27 \\
\hline 3 & 371.208 & 186.108 & 124.407 & W & 2839.5976 & 1420.3025 & 947.2041 & 26 \\
\hline 4 & 499.303 & 250.155 & 167.106 & K & 2653.5183 & 1327.2628 & 885.1776 & 25 \\
\hline 5 & 614.330 & 307.668 & 205.448 & D & 2525.4234 & 1263.2153 & 842.4793 & 24 \\
\hline 6 & 727.414 & 364.211 & 243.143 & L & 2410.3964 & 1205.7018 & 804.1370 & 23 \\
\hline 7 & 840.498 & 420.753 & 80.837 & L & 2297.3123 & 1149.1598 & 766.4423 & 22 \\
\hline 8 & 968.593 & 484.800 & 323.536 & K & 2184.2283 & 1092.6178 & 728.7476 & 21 \\
\hline 9 & 1082.636 & 541.822 & 361.550 & $\mathrm{~N}$ & 2056.1333 & 1028.5703 & 686.0493 & 20 \\
\hline 10 & 1181.704 & 591.356 & 394.573 & v & 1942.0904 & 971.5488 & 648.0350 & 19 \\
\hline 11 & 1238.726 & 619.866 & 413.580 & G & 1843.0219 & 922.0146 & 615.0122 & 18 \\
\hline 12 & 1366.821 & 683.914 & 456.278 & K & 1786.0005 & 893.5039 & 596.0050 & 17 \\
\hline 13 & 1437.858 & 719.433 & 479.957 & A & 1657.9055 & 829.4564 & 553.3067 & 16 \\
\hline 14 & 1508.895 & 754.951 & 503.636 & A & 1586.8684 & 793.9378 & 529.6276 & 15 \\
\hline 15 & 1565.916 & 783.462 & 522.644 & G & 1515.8313 & 758.4193 & 505.9486 & 14 \\
\hline 16 & 1694.011 & 847.509 & 565.342 & K & 1458.8098 & 729.9085 & 486.9415 & 13 \\
\hline 17 & 1765.048 & 883.028 & 589.021 & A & 1330.7148 & 665.8611 & 444.2431 & 12 \\
\hline 18 & 1864.117 & 932.562 & 622.044 & v & 1259.6777 & 630.3425 & 420.5641 & 11 \\
\hline 19 & 1977.201 & 989.104 & 659.738 & L & 1160.6093 & 580.8083 & 387.5413 & 10 \\
\hline 20 & 2091.244 & 1046.126 & 697.753 & $\mathrm{~N}$ & 1047.5252 & 524.2662 & 349.8466 & 9 \\
\hline 21 & 2219.339 & 1110.173 & 740.451 & k & 933.4823 & 467.2448 & 311.8323 & 8 \\
\hline 22 & 2318.407 & 1159.707 & 773.474 & v & 805.3873 & 403.1973 & 269.1340 & 7 \\
\hline 23 & 2419.455 & 1210.231 & 807.156 & $\begin{array}{l}T \\
\end{array}$ & 706.3189 & 353.6631 & 236.1112 & 6 \\
\hline 24 & 2534.482 & 1267.745 & 845.499 & D & 605.2712 & 303.1392 & 202.4286 & 5 \\
\hline 25 & 2665.522 & 1333.265 & 889.179 & M & 490.2443 & 245.6258 & 164.0863 & 4 \\
\hline 26 & 2764.591 & 1382.799 & 922.202 & v & 359.2038 & 180.1055 & 120.4061 & 3 \\
\hline 27 & 2878.634 & 1439.820 & 960.216 & $\mathrm{~N}$ & 260.1353 & 130.5713 & 87.3833 & 2 \\
\hline 28 & & & & $\begin{array}{l}\text { Q- } \\
\text { nidated }\end{array}$ & 146.0924 & 73.5498 & 49.3690 & 1 \\
\hline
\end{tabular}

\begin{tabular}{|c|c|c|c|c|c|c|c|c|}
\hline \#1 & $b(1+)$ & $b(2+)$ & $b(3+)$ & Seq. & $y(1+)$ & $y(2+)$ & $y(3+)$ & \#2 \\
\hline 1 & 72.044 & 36.526 & 24.686 & A & & & & 28 \\
\hline 2 & 185.128 & 93.068 & 62.381 & L & 2839.598 & 1420.302 & 947.204 & 27 \\
\hline 3 & 371.208 & 186.108 & 124.407 & w & 2726.514 & 1363.760 & 909.509 & 26 \\
\hline 4 & 499.303 & 250.155 & 167.106 & K & 2540.434 & 1270.721 & 847.483 & 25 \\
\hline 5 & 586.335 & 293.671 & 196.116 & s & 2412.339 & 1206.673 & 804.785 & 24 \\
\hline 6 & 699.419 & 350.213 & 233.811 & L & 2325.307 & 1163.157 & 775.774 & 23 \\
\hline 7 & 812.503 & 406.755 & 71.506 & L & 2212.223 & 1106.615 & 738.079 & 22 \\
\hline 8 & 940.598 & 470.803 & 314.204 & K & 2099.139 & 1050.073 & 700.385 & 21 \\
\hline 9 & 1054.641 & 527.824 & 352.218 & $\mathrm{~N}$ & 1971.044 & 986.026 & 657.686 & 20 \\
\hline 10 & 1153.709 & 577.358 & 385.241 & V & 1857.001 & 929.004 & 619.672 & 19 \\
\hline 11 & 1210.731 & 605.869 & 404.248 & G & 1757.933 & 879.470 & 586.649 & 18 \\
\hline 12 & 1338.826 & 669.916 & 446.947 & K & 1700.911 & 850.959 & 567.642 & 17 \\
\hline 13 & 1409.863 & 705.435 & 470.626 & A & 1572.816 & 786.912 & 524.944 & 16 \\
\hline 14 & 1480.900 & 740.954 & 494.305 & A & 1501.779 & 751.393 & 501.265 & 15 \\
\hline 15 & 1537.921 & 769.464 & 513.312 & G & 1430.742 & 715.875 & 477.586 & 14 \\
\hline 16 & 1666.016 & 833.512 & 556.010 & K & 1373.721 & 687.364 & 458.578 & 13 \\
\hline 17 & 1737.053 & 869.030 & 579.689 & A & 1245.626 & 623.316 & 415.880 & 12 \\
\hline 18 & 1808.091 & 904.549 & 603.368 & A & 1174.589 & 587.798 & 392.201 & 11 \\
\hline 19 & 1921.175 & 961.091 & 641.063 & L & 1103.551 & 552.279 & 368.522 & 10 \\
\hline 20 & 2035.218 & 1018.112 & 679.077 & $\mathrm{~N}$ & 990.467 & 495.737 & 330.827 & 9 \\
\hline 21 & 2106.255 & 1053.631 & 702.756 & A & 876.424 & 438.716 & 292.813 & 8 \\
\hline 22 & 2205.323 & 1103.165 & 735.779 & v & 805.387 & 403.197 & 269.134 & 7 \\
\hline 23 & 2306.371 & 1153.689 & 769.462 & T & 706.319 & 353.663 & 236.111 & 6 \\
\hline 24 & 2421.398 & 1211.203 & 807.804 & D & 605.271 & 303.139 & 202.429 & 5 \\
\hline 25 & 2552.438 & 1276.723 & 851.484 & M & 490.244 & 245.626 & 164.086 & 4 \\
\hline 26 & 2651.507 & 1326.257 & 884.507 & v & 359.204 & 180.106 & 120.406 & 3 \\
\hline 27 & 2765.550 & 1383.278 & 922.521 & $\mathrm{~N}$ & 260.135 & 130.571 & 87.383 & 2 \\
\hline 28 & & & & $\begin{array}{l}\text { Q- } \\
\text { nidated }\end{array}$ & 146.092 & 73.550 & 49.369 & 1 \\
\hline
\end{tabular}




\section{Figure 3}

(A) Alignment of nucleotide sequences of cloned cDNAs encoding the biosynthetic precursors of DRS-CA-1 and DRS-DU-1. (B) Alignment of open-reading frame sequences of DRS-CA-1, DRS-DU-1 and other dermaseptin peptides characterized from other species

(DRS-PS1 from P. sauvagii (accession No. P24302), DRS-H2 from P. hypochondrialis (accession No. P84597) and DRS-B4 from P. bicolor (accession No. P81486)). Conserved amino acid residues are shaded in black. 
1

A) $\quad \begin{aligned} & \mathrm{DRS}-\mathrm{CA}-1 \\ & \mathrm{DRS}-\mathrm{DU}-1\end{aligned}$

(1)

(1) ATGGCTTTCA 51

(51)

DRS-CA-1

DRS-DU-1

\section{TTCTCTTTC}

TTCССTTTCC

101

DRS-CA-1

(101)

ATr

DRS-DU-1

DRS-CA-1

DRS-DU-1

DRS-CA-1

DRS-DU-1

TACTGATATGGTAAATCAAGGAGAGCAATAAAGTTAAGAAAATGTAAATT

TACTGATATGGTAAATCAAGGAGAGCAATAAAGTTAAGAAAATGTAAATC 251

DRS-CA-1

DRS-DU-1

(251)

(251)

TCATAACTCTAAGGAGCACAATT 301

CTCTAAGGAGCACAAA AAGAAATCTCTTTTCCTTGTACTATTCCTTGGATTGGT

50

DRS-CA-1

(297)

(301)

DRS-DU-1

1

B) $\mathrm{DRS}-\mathrm{CA}-1$

(1) MAFLKKSLFLVLFLGLVSLSICEEEKRENEEKENQEDDEQSEEKRALWKD

DRS-DU-1

(1)

DRS-PS1

(1)

MAFIKKSLFLVLFLGLVSLSICE CEEEKRENEEENNQEDDEQSEEKRALWKS

DRS-H2

(1)

MAFLKKSLFLVLFLGLISLSF

CEEEKRENEDEEEQEDDEQSEEKRGLWKS

DRS-B4

(1)

MAFLKKSLFLVLFLGLVSLSICE

CEEEKRENEEKENQEDDEQSEEKRALWKD

MAFLKKSLFLVLFLGLVSLSICEEEKRENKDE IEQEDDEQSEEKRALWKD

51

76

DRS-CA-1 (51) LLKNVGKAAGKAVLNKVTDMVNQGEQ

DRS-DU-1 (51) LLKNVGKAAGKAALNAVTDMVNQGEQ

DRS-PS1 (51) LFKNVGKAAGKAALNAVTDMVNQGEQ

DRS-H2 (51) LLKNVGKAAGKAALNAVTDMVNQGEQ

DRS-B4

(51) ILKNVGKAAGKAVLNTVTDMVNQGEQ 


\section{Figure 4}

CD spectra of the four peptides $(100 \mu \mathrm{M})$ in $10 \mathrm{mM}$ ammonium acetate buffer $(\mathrm{A})$ and $50 \%$ TFE ammonium acetate buffer (B). Helical wheel projections [22] of peptides (C), with the arrow indicated the direction of the hydrophobic moments.

All the peptides exhibit random coil structure in the aqueous solution while they are able to form $\alpha$-helical structure in the membrane-mimetic environment. The hydrophobic (yellow), hydrophilic (purple), positively-charged (blue), negatively-charged (red), amide (pink) and small (grey) residues are presented.

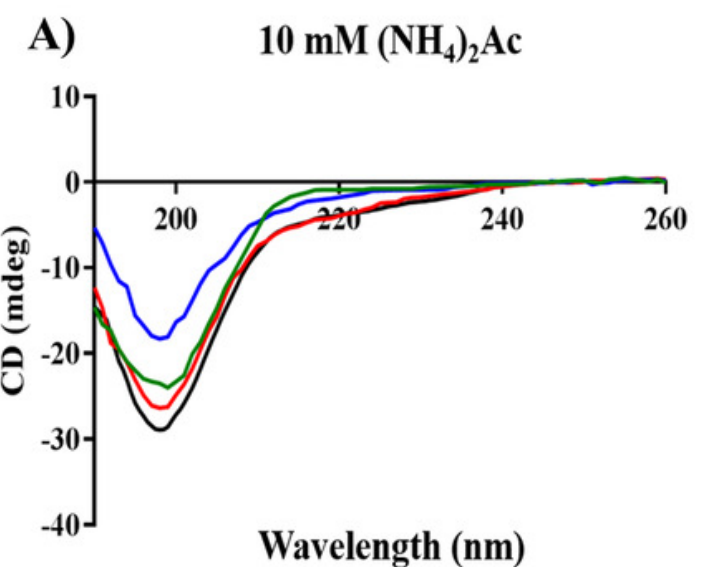

C)

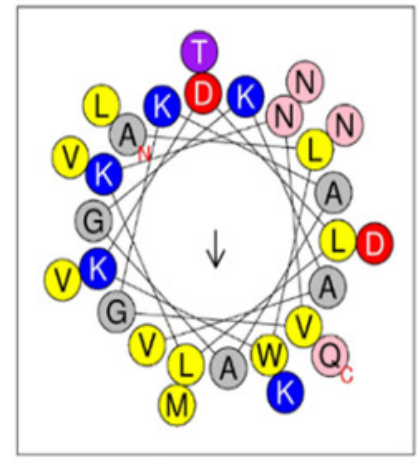

DRS-CA-1

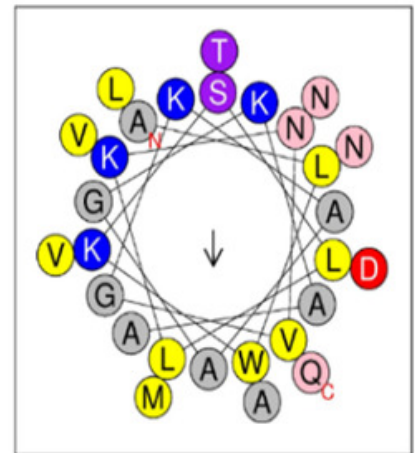

DRS-DU-1

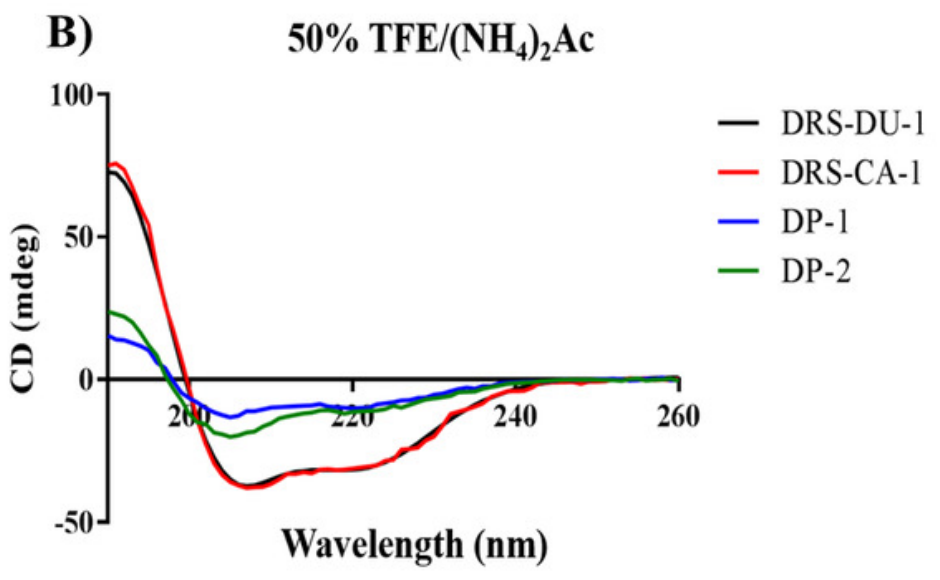

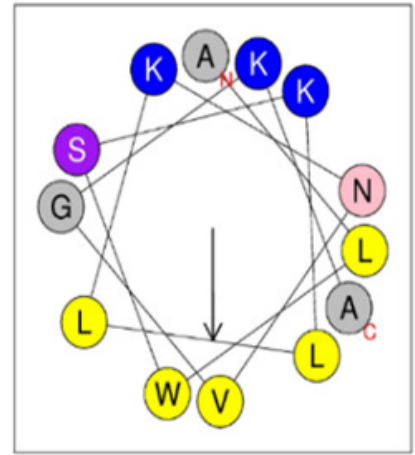

DP-1

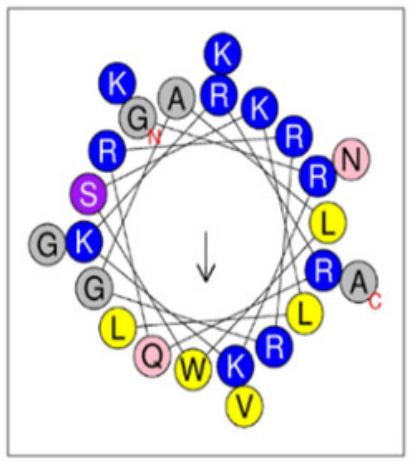

DP-2 